\title{
Natural Convection Boundary Layers Adjacent to Pyrolyzing Surfaces
}

\author{
PATRICK V. FARRELL \\ Department of Mechanical Engineering, The University of Wisconsin-Madison, Madison, WI 53706
}

and

\section{GEORGE S. SPRINGER and CHARLES M. VEST}

\author{
Department of Mechanical Engineering and Applied Mechanics, The University of Michigan, Ann Arbor, MI 48109
}

\begin{abstract}
The temperature, velocity, and concentration distributions were measured in boundary layers adjacent to heated vertical polymethyl methacrylate (PMMA) and wood (Southem Pine) surfaces, and next to a heated, vertical, sintered bronze plate through which propane was injected. The temperature, velocity, and concentration were measured as functions of position and time using thermocouple probes, flow visualization techniques, and heterodyne holographic interferometry, respectively. The measured distributions were compared to the results of a simple model. The agreement between the measured values and the results of the model was generally good and was within the experimental uncertainty.
\end{abstract}

\section{INTRODUCTION}

A problem of current interest is the release of volatile vapors from radiantly heated solids and the subsequent transport of the volatiles in the boundary layer adjacent to the solid. Many workers have studied the problems of solid pyrolysis ([1-5], for example). While most agree that the gas phase reactions are central to the ignition process ([4], for example), few have included this aspect in a generalized way in their calculations of volatile release or their numerical modeling of pyrolysis and ignition. Those studies which have incorporated gas phase transport have limited it to one-dimensional flows [3], or steady similaritytype flows [5], or have used an unsteady approximate method [4].

This investigation addressed the study of the characteristics of the injection and subsequent diffusion of a volatile into a laminar, natural convection boundary layer adjacent to a vertical slab or surface. This configuration was chosen because of its prevalence in studies of solid fuel pyrolysis. This study concentrates on low temperature pyrolysis as a limiting case for both experimental measurements and numerical modeling. While heating rate and solid temperature are significant factors in volatile formation, it is anticipated that prior to gas phase chemical reaction, these variables affect the gas phase temperature and concentration distribution only through changes in the boundary conditions at the solidgas interface.

The first objective of this effort was to develop an experimental technique which would allow accurate measurement of the variables of interestnamely temperature, velocity, and concentration in the low temperature pyrolysis range. The sec- 
ond objective was to measure these variables as accurately as possible to allow comparison with analytic results. Finally, a third objective was to develop a simple model of this problem and to compare the results obtained with the data. It is anticipated that as data for higher pyrolysis rates prior to gas phase reactions become available, the model described will continue to work well with suitably specified boundary conditions. The experimental technique was described elsewhere [6]; therefore, emphasis is placed on the second objective.

The problem of volatile ejection and diffusion from a heated surface, if considered in a simple geometry prior to the large gas phase heat release accompanying ignition, is similar to the problem of combined heat and mass transfer in natural convection, which has received considerable attention. Ostrach [7] has recently reviewed the work in this field. Even in the general heat and mass transfer literature there is little available data which can be used for comparison with numerical work. Further, most extant numerical efforts involve integral or similarity analysis, which do not always allow for appropriate statement of the physics of the problem. This effort provides data for study of the preignition solid pyrolysis problem in the gas phase, as well as for the combined heat and mass transfer problem. In addition, a nonsimilar numerical model is developed and compared to the measurements.

\section{EXPERIMENTS}

The problem studied consisted of a vertical flat slab of material. At some time $t_{0}$, the slab began being irradiated by a high temperature source. As the slab heated, pyrolysis occurred, liberating vapors which migrated to the surface of the slab. The vapors emitted from the slab formed a concentration boundary layer in the ambient fluid (air), while the heated surface of the slab initiated a thermal one.

Experiments were performed to measure the temperature, velocity, and concentration distributions in the air adjacent to the heated vertical surface. The vapors used in the tests were propane, introduced through a sintered bronze plate, and hydrocarbons issuing from heated polymethyl methacrylate (PMMA) and Southern Pine wood plates. The propane injection provided a controllable force flow of heavier-than-air fluid through the surface. The heated PMMA and Southern Pine specimens released heavier- and lighter-than-air vapors, respectively. The amount of vapor released by the solid specimens depended on the internal temperature distribution of the solid $[8,24]$.

Temperatures were measured using fine wire thermocouples. Velocities were measured using a flow visualization technique. The concentrations of vapors were measured using heterodyne holographic interferometry.

\section{Test Section}

The test section consisted of a flat vertical specimen, which was radiantly heated by suspending it between two opposing electrical heaters (Fig. 1). Three types of specimens were used in the tests: PMMA (Rohm and Haas Plexiglas G), Southern Pine (standard construction grade with a nominal moisture content of $6 \%$ ), and a sintered bronze plate. The specimen dimensions are given in Fig. 1. One thickness of sintered bronze $(0.3175 \mathrm{~cm})$, one thickness of PMMA $(2.54 \mathrm{~cm})$, and two thicknesses of Southern Pine $(0.3175$ and $2.54 \mathrm{~cm})$ were tested. Five copper-constantan thermocouples were imbedded in the PMMA and Southern

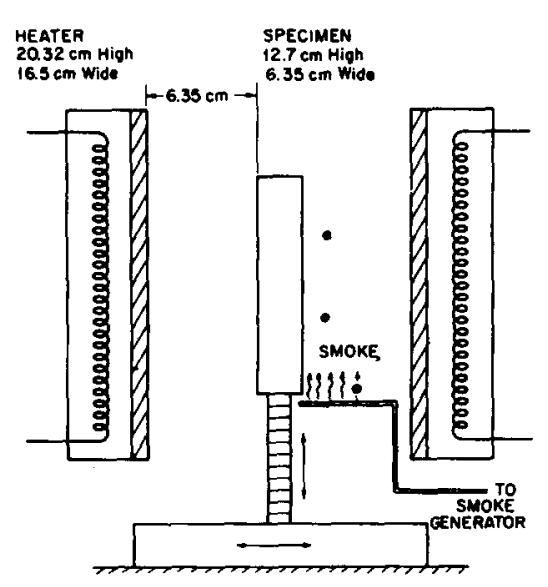

Fig. 1. Schematic of apparatus used with PMMA and Southern Pine specimens: (o) represents thermocouples. 
Pine specimens to determine the surface temperature and uniformity. The sintered bronze plate was mounted opposite a single radiant heater and was attached to an aluminum chamber which served as a plenum for the propane. The propane flow rate was measured using two micromanometers attached to the piping system.

Each heater consisted of Chromel wire coils mounted on an asbestos plate enclosed in a steel chamber. The maximum power output of each heater was $1270 \mathrm{~W}$. The voltage to the heater was controlled by a variable transformer. The temperature distribution across the heater faceplate was measured by thermocouples and also by an infrared thermometer. The temperature variation across the surface of the heater was found to be less than about $2 \%$. The experiments were performed with two values of incident radiation ( 3180 and $1140 \mathrm{~W} / \mathrm{m}^{2}$ ), which was measured with a Gardon heat flux detector. The variation of incident radiation flux across the surface of the specimen was less than $5 \%$ of the measured value.

\section{Temperature Measurements}

The temperature of the vapor-air mixture in the boundary layer was measured by inserting 0.0762-mm-diameter unshielded copper-constantan thermocouples into the space between the specimen and the heaters. The distance of the thermocouples from the specimen surface could be adjusted to within $0.0127 \mathrm{~mm}$. Two thermocouples were mounted on a traversing stage, one at a height of $5.4 \mathrm{~cm}$ from the leading (lower) edge of the specimen, and the other at a height of 8.9 $\mathrm{cm}$. Measurements were taken at seven different locations normal to the wall, at $2.5 \mathrm{~min}$ intervals. Prior to the measurement, the stage was translated to allow the two thermocouples to touch the wall. This was visually checked using a Gaertner cathetometer. The thermocouples were then withdrawn to the prescribed position, using the micrometer screw on the stage to measure displacement. For each specimen, the temperature was measured at the two vertical positions every 2.5 $\mathrm{min}$. The thermocouple readings were corrected for the effects of conduction, convection, and radiation. Five thermocouples were embedded in the solid. The temperatures provided by these thermocouples were extrapolated to the surface. These extrapolated values were compared with corrected surface temperature measurements made by the gas phase thermocouples. An average of the values was used as the estimated surface temperature value. Using the method of least squares, a third-order polynomial was fitted to the temperature measured (at a given time) at each of the seven locations perpendicular to the wall and the estimated surface temperature. In the subsequent analysis this polynomial was used to calculate the temperature as a function of position. Ambient temperature was continuously measured as well. In an effort to reduce all room drafts, the ventilation system was blocked, resulting in an ambient temperature of $10^{\circ} \mathrm{C}$ for these tests.

The accuracies of the temperature measurements were assessed by measuring the air temperature adjacent to a vertical, heated, isothermal aluminum surface. The temperatures measured with the thermocouples were compared to (a) the temperature measurements made using holographic interferometry [9], (b) values predicted by Ostrach's similarity solution [10], and (c) the results of the model developed in this investigation. The comparisons, given in Fig. 2, show good agreement between the measured and calculated temperatures.

\section{Velocity Measurements}

The velocities of the vapor-air mixture between the specimen and the heater were measured by introducing titanum tetrachloride $\left(\mathrm{TCl}_{4}\right)$ into the mixture through a perforated 18 gauge hypodermic needle placed perpendicular to the test specimen near the bottom edge of the specimen. One end of the needle was sealed, while the other end was connected to the $\mathrm{TCl}_{4}$ reservoir. $\mathrm{A}$ stopcock was opened for short periods of time $(\sim 1 \mathrm{~s})$, allowing small amounts of $\mathrm{TCl}_{4}$ to be introduced into the mixture through holes drilled in the needle. The $\mathrm{TCl}_{4}$ created puffs of smoke which were entrained in the boundary layer adjacent to the specimen. The velocity of the smoke puffs, and hence the velocity of the mixture, were determined by high speed photography. 

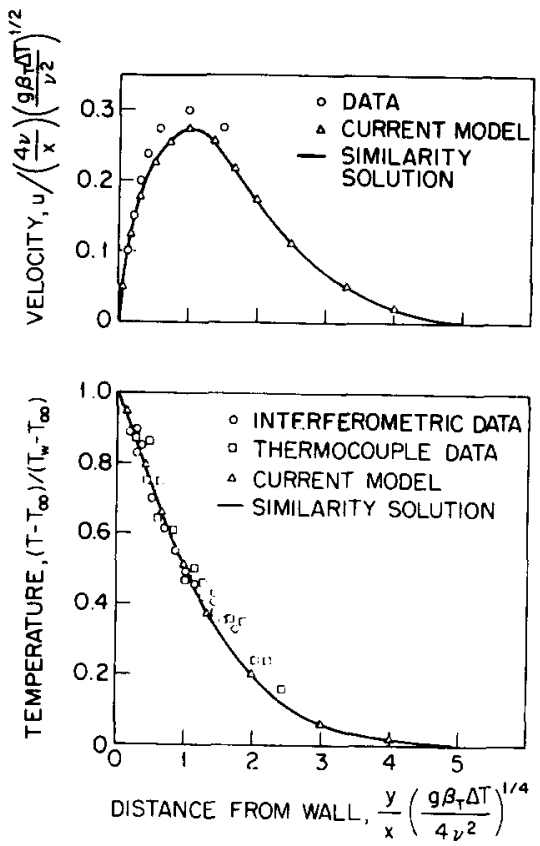

Fig. 2. The steady state velocity and temperature distributions adjacent to heated vertical aluminum surface. Similarity solu tions given by Ostrach [10].

Using the method of least squares a fourth-order polynomial was fitted to the data, providing the velocity as a function of position from the surface. In order to assess the accuracy of the test method, velocity distributions were observed adjacent to a vertical, heated, isothermal aluminum plate. The data were compared to the results of Ostrach's similarity solutions and to the results obtained by the present model (Fig. 2). There is good agreement between the measured and calculated velocities.

\section{Concentration Measurements}

The concentration of the vapor in the boundary layer was measured by the technique of heterodyne holographic interferometry. Since details of the apparatus and experimental procedures are described elsewhere [6], only the essential features of the method are outlined here.

A laser beam was split into three parts, called the object beam, reference beam 1 , and reference beam 2 (Fig. 3). Reference beam 1 passed through two acoustooptic modulators which could be used

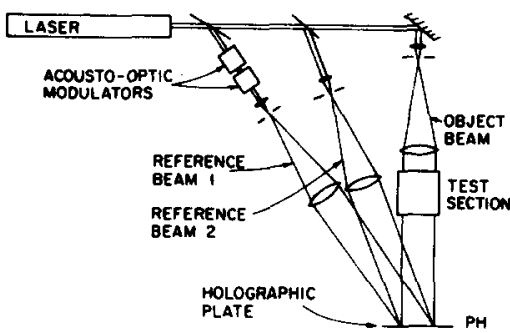

Fig. 3. The optical system used in heterodyne holographic interferometry.

to shift the light frequency. The object beam and both reference beams were expanded, collimated, and directed toward a holographic plate mounted at PH. A first exposure was made using reference beam 2 and the object beam while the specimen plate and ambient air (denoted as component 2 of the mixture) were at room temperature. During this exposure, reference beam 1 was blocked. A second exposure was taken using reference beam 1 and the object beam while the specimen plate was heated and component 1 was injected into the boundary. During this exposure reference beam 2 was blocked. After the second exposure the holographic plate was developed, replaced in its original position, and illuminated by both reference beams, thereby holographically reconstructing the two object waves. These reconstructed light waves interfered to form alternating light and dark fringes on a ground glass screen placed behind the hologram. These fringes were interpreted to determine the refractive index distribution (and consequently the density distribution) in the boundary layer.

In conventional holographic interferometry, refractive index distributions are determined by visually measuring the positions of the centers of bright and dark fringes. Fractional fringe measurements-that is, interpolations between fringe centers-generally are difficult and inaccurate. Heterodyne holographic interferometry is a technique whereby measurements are made electronically which are equivalent to very accurate fractional fringe measurements. To accomplish this, the holographic plate was exposed, developed, and replaced in its original position as described in the preceding paragraph. It was illuminated by both reference beams while the temporal fre- 
quency of reference beam 1 was shifted by 50 $\mathrm{kHz}$ relative to that of reference beam 2 by the two acoustooptic modulators.

Two photodiodes were placed in the plane of the fringe pattern. The outputs of the two photodiodes oscillated at $50 \mathrm{kHz}$ but were out of phase with each other. The phase difference between their outputs was equivalent to the fringe shift between the locations of the two detectors. With this technique phase shifts as small as $0.3^{\circ}$ have been detected [11], in contrast to the method of conventional interferometry, where phase shifts of $180^{\circ}$ can be measured accurately.

From the Lorenz-Lorentz equation and the equation of state for ideal gases [12] the concentration of component 1 in a binary mixture can be calculated using the expression

$$
\begin{aligned}
c= & \frac{r_{1}}{r_{1}+r_{2}}=\frac{2 S \lambda R T}{3 l P_{\infty}\left(A_{1}-A_{2}\right)}-\frac{A_{2}}{A_{1}-A_{2}} \\
& \times\left[1-\frac{T}{T_{\infty}}\right]
\end{aligned}
$$

where $r_{1}$ and $r_{2}$ are the number of moles and $A_{1}$ and $A_{2}$ are the molar refractivities of the components 1 and 2, $T_{\infty}$ is the temperature at which the first of the two exposures is taken (ambient temperature). $P_{\infty}$ is the atmospheric pressure, $R$ is the universal gas constant, $T$ is the local temperature of the mixture at the time when the second exposure is taken, $\lambda$ is the wavelength of the laser light, and $l$ is the path length of light across the test surface. $S$ is the fringe number, which is determined from the measured phase shift $P_{s}\left(S=P_{s} / 360\right)$. The experimentally determined values of $S$ must be corrected for refractive and edge effects. Refractive corrections were necessary for strong gradients of index of refraction (density). In this experiment, only the propane injected through the sintered bronze plate produced such gradients. A computer code developed by Cha and Vest [13] was used to correct for the bending of the object wave due to the index of refraction gradients.

The edge effects were accounted for on all specimens using the model of Hauf and Grigull [14] for the "spillover" of a boundary layer. This model assumes that the edge effects are due to the addition of a layer of fluid in the shape of a quarter-cylinder, at each edge of the plate. A corrected reading subtracts the effects of these quarter-cylinders.

The molar refractivities of propane and of the vapors issuing from Southern Pine and PMMA were estimated using the expression [12]

$A_{\text {mix }}=\frac{r_{1}}{r_{1}+r_{2}} A_{1}+\frac{r_{2}}{r_{1}+r_{2}} A_{2}$

and

$A_{n}=\nu_{\alpha} A+\nu_{\beta} A_{\beta}+\nu_{\gamma} A_{\gamma}$,

where $A_{\mathrm{mix}}$ and $A_{n}$ are the molar refractivities of a mixture of compounds and a single compound, respectively. $A_{\alpha}, A_{\beta}, A_{\gamma}$ are the molar refractivities and $\nu_{\alpha}, \nu_{\beta}, \nu_{\gamma}$ are the number of atoms of the elements present in the compound. The molar refractivity values used in this work are given in Table 1.

Subsequent to the initiation of these experiments, updated values of molar refractivities have been published by Gardiner et al. [27]. For the case of propane and that of water, the updated values change the propane concentration values by about $3 \%$, and those of the wood vapor by about $1 \%$. The molar refractivity for MMA vapor listed in Table 1 was calculated using Eq. (3) and the values given in [12]. The elemental molar refractivities given by the results of Gardiner et al. [27] were combined as in Eq. (3) to compare the results produced in this way with directly listed molar refractivities of known compounds. The values in Table 2 of Ref. [27] worked fairly well in this comparison. The molar refractivity calculated for MMA from the values in Table 2 of Ref. [27] was only slightly different from that cited herein. As a consequence of these considerations, the differences in the values of the molar refractivities utilizing Ref. [27] and those utilizing the values in Table 1 and the resulting effect on the concentration distributions are considered negligible for the purposes of these experiments. 
TABLE 1

Molar Refractivities $(A)$ Used in the Calculations

\begin{tabular}{|c|c|c|c|c|c|}
\hline Material & Compound ${ }^{a}$ & $\begin{array}{c}\text { Mole } \\
\text { Fraction } b\end{array}$ & Element & $\begin{array}{l}A \text { of Element } \\
\text { or Compound } \\
\left(\mathrm{cm}^{3} / \text { mole }\right)\end{array}$ & $\begin{array}{c}A_{\operatorname{mix}} \text { for } \\
\text { Vapord } \\
\left(\mathrm{cm}^{3} / \text { mole }\right)\end{array}$ \\
\hline Southern & $\mathrm{H}_{2} \mathrm{O}$ & 0.854 & & 3.68 & 3.568 \\
\hline \multirow[t]{6}{*}{ Pine } & $\mathrm{H}_{2}$ & 0.0038 & $2 \mathrm{H}$ & 1.02 & \\
\hline & $\mathrm{CO}$ & 0.0684 & & 2.609 & \\
\hline & $\mathrm{CO}_{2}$ & 0.0704 & & 3.491 & \\
\hline & $\mathrm{CH}_{4}$ & 0.0013 & & 3.446 & \\
\hline & $\mathrm{C}_{2} \mathrm{H}_{4}$ & 0.0015 & & 5.578 & \\
\hline & $\mathrm{C}_{2} \mathrm{H}_{6}$ & 0.0002 & & 5.874 & \\
\hline \multirow[t]{3}{*}{ PMMA } & $\mathrm{C}_{5} \mathrm{H}_{8} \mathrm{O}_{2}$ & 1.00 & $5 \mathrm{C}$ & 2.11 & 22.73 \\
\hline & & & $8 \mathrm{H}$ & 1.02 & \\
\hline & & & 20 & 2.01 & \\
\hline \multirow{2}{*}{ Propane } & $\mathrm{C}_{\mathbf{3}} \mathrm{H}_{8}$ & 1.0 & $3 \mathrm{C}$ & 2.11 & 14.49 \\
\hline & & & $8 \mathrm{H}$ & 1.02 & \\
\hline
\end{tabular}

a The compounds present in the vapors emitted from Southern Pine are from Refs. $[1,8]$.

$b$ The mole fraction of compounds are from [1,8] for Southern Pine and from [24] for PMMA.

c $A$ for elements from [12], $A$ for compounds from [23].

d $A_{\mathrm{mix}}$ of the volatiles was calculated using Eq. (2).

\section{Experimental Procedure}

In each experiment, the heaters were allowed to warm up for $2 \mathrm{~h}$. Radiation shields were placed between the heaters and the specimen holder to allow careful, unheated positioning of the specimen. The specimen was then placed in the specimen holder, the radiation shields were removed, and the appropriate measurements were made. The temperature and velocity of the vapor-air mixture and the concentration of the vapors were measured in sequence each test being performed with a different, but nominally identical specimen.

\section{RESULTS}

As stated in the introduction, the primary objective of this investigation was the experimental study of the preignition behavior of boundary layers adjacent to radiantly heated solid surfaces. The value of the heat flux and resulting surface temperature on a specimen was chosen to simulate the condition of the initial heating of a solid and beginning of the vapor emission process. The resulting surface temperatures are quite low by conventional pyrolysis standards. However, several calculations were performed to assure, as nearly as possible, that the expected volatiles were indeed being emitted.

In the case of the wood specimens, Stamm [8] and Williams [25] indicate that thermal degradation can occur in wood specimens at temperatures below $200^{\circ} \mathrm{C}$. While the majority of vapor produced at these temperatures is water, the above references indicate other products should be expected. Mass loss in cellulosic materials is often modeled as a first-order Arrhenius equation. By using the constants in [25] a rough calculation shows that the wall concentration of volatiles measured agrees well with a predicted value based on the calculated mass flux for the measured wall temperature.

The PMMA specimens are also heated to a relatively low value of the surface temperature, compared with the range in which they produce large amounts of vapor. However, even at the surface temperatures used, some thermal effects should be expected. A rough calculation using the accepted first-order Arrhenius equation and the constants for PMMA from [24] indicates that the measured wall concentration values agree well with values calculated from a predicted mass flux based on the measured wall temperatures.

The data generated are presented in this sec- 
TABLE 2

Magnitude of Estimated Most Probable Experimental Errors (\%)

\begin{tabular}{lccc}
\hline Material & Velocity & Temperature & Concentration \\
\hline Aluminum & 19 & 3 & \\
Sintered bronze & & & 3 \\
plate & 19 & 2 & 23 \\
PMMA & 19 & 3 & 26 \\
Southern Pine & & 4 & \\
\hline
\end{tabular}

tion. Since considerable data were taken, only typical results are discussed here. A full description of the experimental results is given in [9]. In addition to presenting the data, comparisons between the data and the results of a model (developed in the Appendix) are also shown.

A detailed analysis was made of the experimental errors. The errors (summarized in Table 2) should be borne in mind when examining and interpreting the data.

\section{Sintered Bronze Plate}

The temperature, velocity, and propane concentration were measured $8.9 \mathrm{~cm}$ from the leading (bottom) edge of a vertical, sintered bronze plate, with propane being injected through the plate uniformly at a rate of $\dot{m}_{\mathrm{w}}=0.0628 \mathrm{~g} / \mathrm{s}$. Measurements were made with an incident radiant heat flux of $3180 \mathrm{~W} / \mathrm{m}^{2}$ (surface temperature of $55.6^{\circ} \mathrm{C}$ ), and with the plate not heated. The data are presented in Fig. 4. Note that in the case of the unheated plate, the mean velocity was downward because propane is heavier than air.

The temperature, velocity, and concentration distributions also were calculated using the model for the experimentally determined values of the wall concentration ( 0.32 moles/mole) and the injection velocity normal to the surface $(0.602$ $\mathrm{cm} / \mathrm{s})$. The mixture properties were assumed to be those of pure air. The calculations required knowledge of the molar diffusivity of propane in air. The diffusivity $D$ was estimated through the use of the Schmidt number $(S c=\nu / D)$. For a dilute propane-air mixture, the Schmidt number is nearly constant and has a value of 1.51 [15] .
This value of the Schmidt number and the known value of the kinematic viscosity of air, $\nu$, were used to calculate $D$. The Prandtl number was also calculated for the conditions of the experiments and was found to be nearly constant (Pr = 0.72 ).

The results of the model are also included in Fig. 4. The measured and calculated temperatures and concentrations agree within $2 \%$. The measured and calculated velocities agree within $10 \%$ for the heated test. There is a discrepancy between the predictions and data for the case of an unheated plate with propane injection. This discrepancy was attributed to the experimental apparatus, in which it proved difficult to eliminate all extraneous air currents, yet allow for exhaust of the toxic $\mathrm{HCl}$ vapor. As the data show, this only affected the results in the case of downward flow at distances far from the surface.

\section{PMMA and Southern Pine Plates}

Temperatures and vapor concentrations were measured near PMMA (2.54 cm thick) and Southern Pine ( $2.54 \mathrm{~cm}$ thick) plates exposed to radiant heat fluxes for different lengths of time. Temperature and concentration were measured along two lines, one $5.4 \mathrm{~cm}$ and the other $8.9 \mathrm{~cm}$ from the lower edge of the plate. Samples of the data are shown in Figs. 5 and 6.

The temperatures, velocities, and concentrations were calculated using the model at the same location where measurements were made. The calculations were performed using the experimentally determined values of the wall temperature, ambient temperature, and vapor concentration at the surface $\left(c_{w}\right)$. The normal velocity at the wall was evaluated from the known value of $c_{w}$ [Eq. (A.9)]. The Schmidt number was estimated by the following relation recommended for dilute air-vapor mixtures [15] :

$S C=0.145 M^{0.556}$

where $M$ is the molecular weight of the vapor. In the case of PMMA, the vapor is MMA, which has a molecular weight of 100 . This gives a Schmidt 

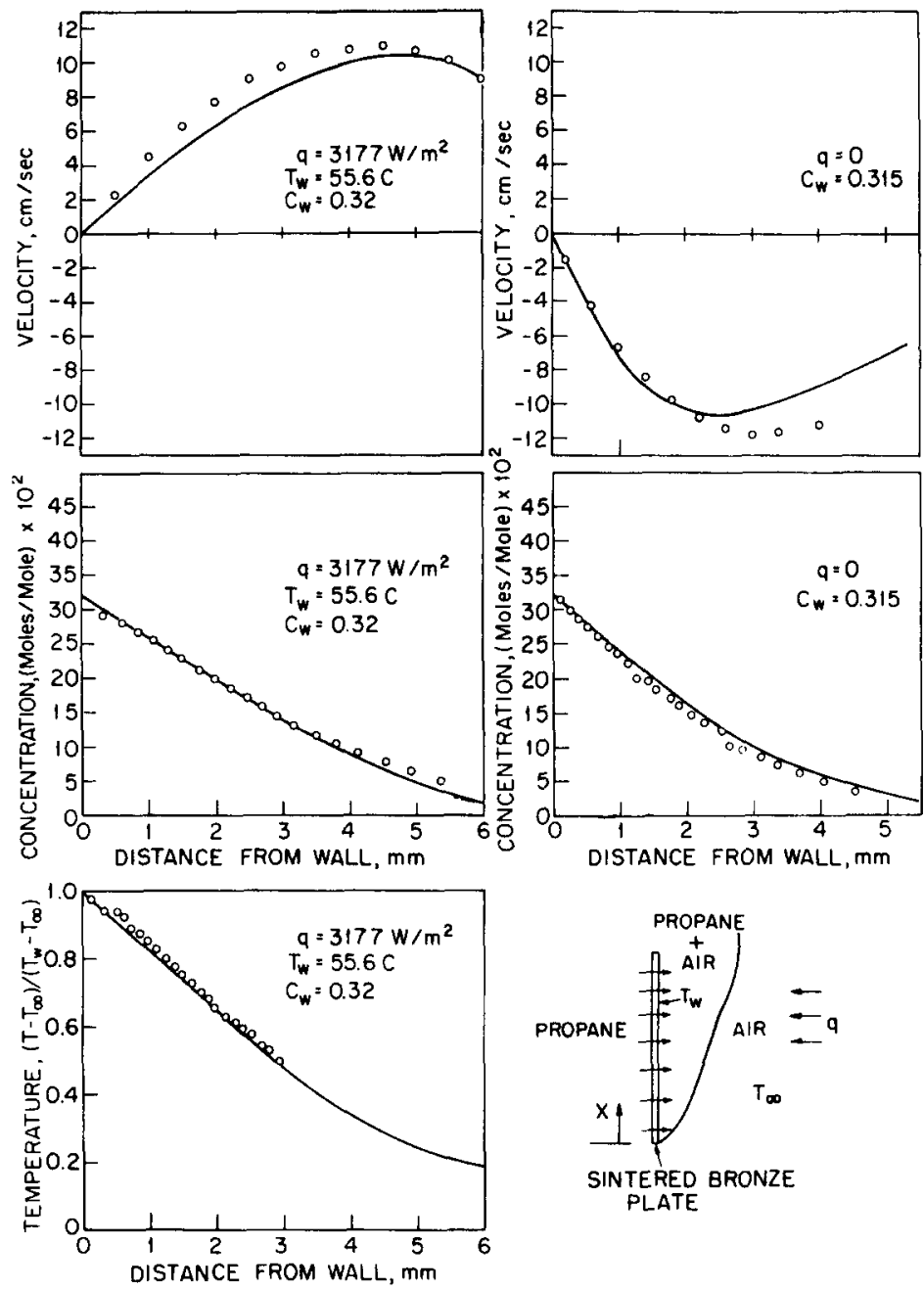

Fig. 4. Propane injected through a sintered bronze plate: $(0)$, data; $(-)$, results of the model $\left(T_{\infty}=20^{\circ} \mathrm{C}, \operatorname{Pr}=0.72, S c=1.51\right)$.

number of 1.88 (here approximated as 2.0) and a diffusivity $D=0.074 \mathrm{~cm}^{2} / \mathrm{s}$.

The vapors issuing from Southern Pine specimens were composed of several different substances. The substances present in the vapor and their concentrations varied throughout the test. When the surface was first exposed to heat most of the vapor issued was water. As the heating progressed, the vapor contained more combustible products. In the calculations, the composition of the vapor was taken to be constant and was assumed to be $85 \%$ (molar) water, $0.8 \% \mathrm{H}_{2}, 6.83 \%$ $\mathrm{CO}, 7.0 \% \mathrm{CO}_{2}, 0.13 \% \mathrm{CH}_{4}, 0.15 \% \mathrm{C}_{2} \mathrm{H}_{4}$, and $0.02 \% \mathrm{C}_{2} \mathrm{H}_{6}[1,8]$. The molecular weight of this mixture is $20.6 \mathrm{~g} / \mathrm{g}$ mole, the Schmidt number is 0.8 [Eq. (4)], and the diffusivity is 0.167 $\mathrm{cm}^{2} / \mathrm{s}$. The Prandtl number was calculated for the conditions of the experiments and was found to be nearly constant at a value of 0.72 .

The results of the model are included in Figs. 5 and 6. For the tests with PMMA specimens, the measured and calculated temperatures, velocities, and concentrations agree within about 4,10 , and $15 \%$, respectively. These agreements are within the accuracies of the measurements. For Southern Pine specimens, the measured and calculated values of the temperatures agree closely $(\sim 3 \%)$. There seems to be a small systematic deviation 

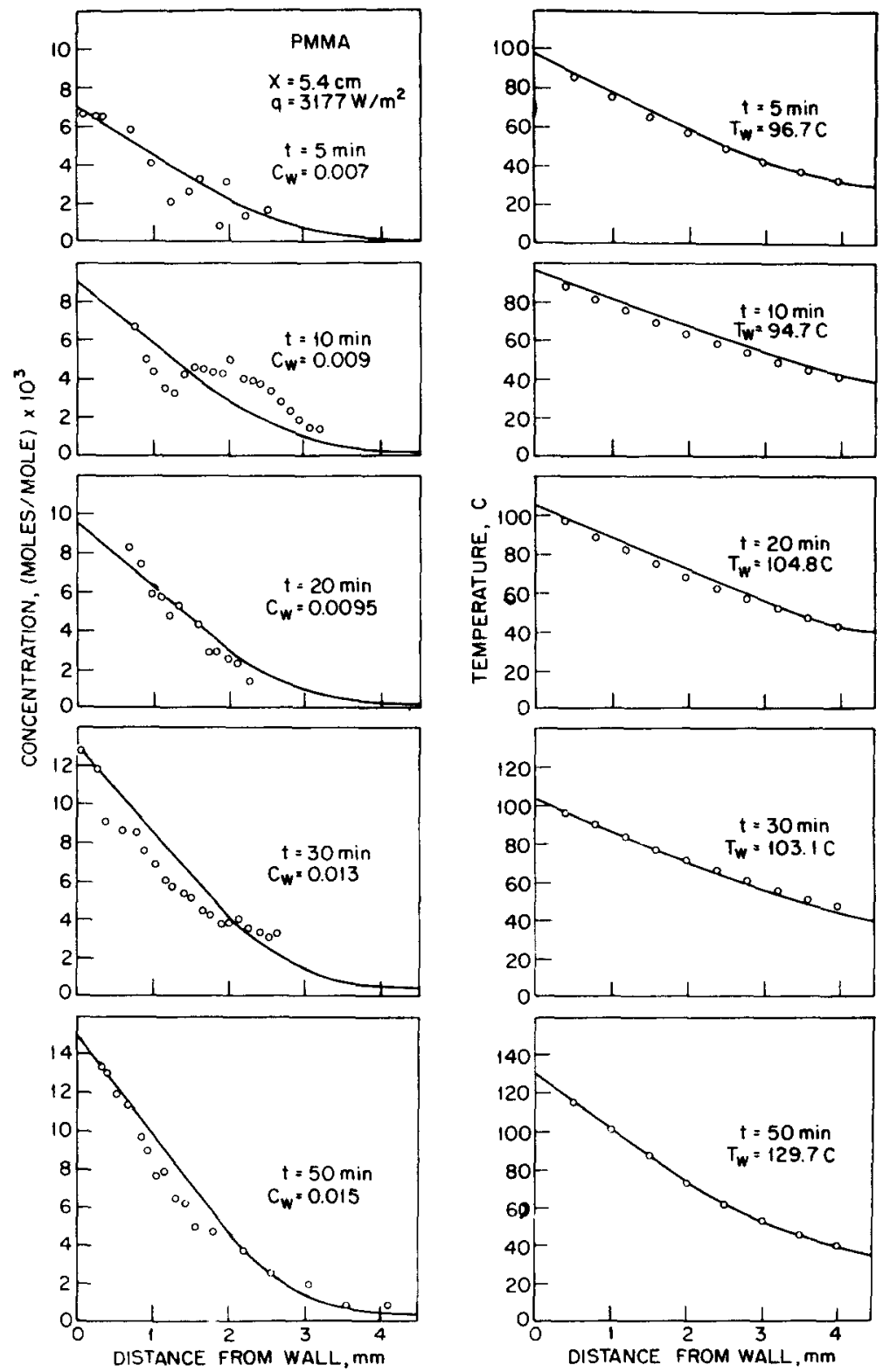

Fig. 5. Vapor concentrations and temperatures adjacent to a heated vertical PMMA Surface: (O), data; $(-)$, results of the model $\left(T_{\infty}=20^{\circ} \mathrm{C}, M=100, \operatorname{Pr}=0.72, S c=2.0\right)$.

between the measured and calculated values of the concentration. This is most likely due to the fact that in the calculation, the molecular weight of the vapor was taken to be constant. In fact, as noted above, the composition of the vapor, and hence the molecular weight of the vapor, varies throughout the test. As time progresses, the vapors contain less water and heavy hydrocarbons and more light hydrocarbons, resulting in a decrease of molecular weight with time. If a decreasing molecular weight were incorporated into the model, a lower concentration prediction would result giving closer agreement with the data. A changing value of the molecular weight of the 

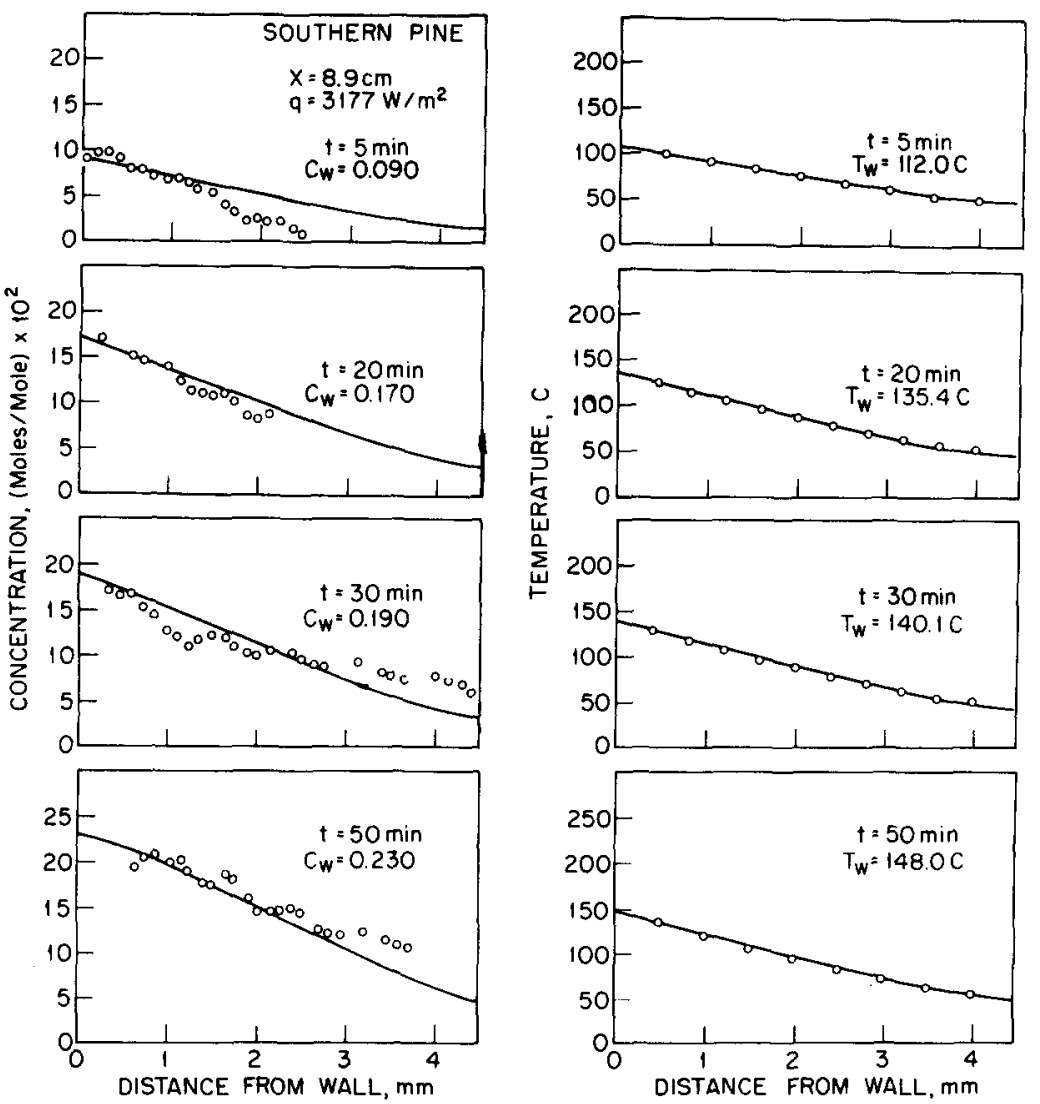

Fig. 6. Vapor concentrations and temperatures adjacent to a heated vertical Southern Pine surface, $2.54 \mathrm{~cm}$ thick: (o), data; $(-)$, results of the model $\left(T_{\infty}=20^{\circ} \mathrm{C}, M=20.6\right.$, $\operatorname{Pr}=0.72, S c=0.8$ ).

vapor could have been incorporated into the numerical solution. This, however, was not done since the variation of composition (and molecular weight) with time was not known with sufficient accuracy. Nevertheless, the calculations based on the assumption of constant molecular weight indicate the trends in the concentration distributions.

In addition to the temperature, velocity, and concentration profile measurements, calculations were made of the Nusselt and Sherwood numbers, based on the total height of the plate. The computer model also calculated these values. While objectives of this study did not include consideration of these parameters, they are instructive for evaluating the model and limited comparison with previous work. Consequently, these values will be discussed only briefly. In general, the meas- ured and predicted values of Nusselt and Sherwood agree fairly well over the range of experiments, being usually within $10 \%$, and never more than $20 \%$ different. Considering the accuracy of the data, these values are as good or better than could be expected.

Comparisons can be made of the parameters $N u_{\mathrm{L}} / G r_{\mathrm{L}}{ }^{1 / 4}$ and $S h_{\mathrm{L}} / G r_{\mathrm{L}}{ }^{1 / 4}$ to the work of Gebhart and Pera [16] and Callahan and Marner [17] for the sintered bronze plate, where the assumption of constant wall temperature is justified. For the $N=0$ case

$\left(N=\frac{\beta_{c}\left(c_{\mathrm{w}}-c_{\infty}\right)}{\beta_{t}\left(T_{\mathrm{w}}-T_{\infty}\right)}\right.$ as in [16] and [17]),

the experiment yields steady state values of $\overline{N u}_{\mathrm{L}} / G r_{\mathrm{L}}{ }^{1 / 4}=0.481$, the predicted value is 
0.473 , and the value graphed by Callhan and Marner is approximately 0.52 . Gebhart and Pera graph a value of $N u_{x} / G r_{x}{ }^{1 / 4}$ of about 0.36 . Further comparisons with either the similarity solutions of Gebhart and Pera or the constant wall temperature numerical solutions of Callahan and Marner are not possible due to differences in the applied boundary conditions.

\section{CONCLUDING REMARKS}

In order to predict preignition volatile generation and distribution accurately, understanding the thermal and concentration boundary layers adjacent to a pyrolyzing solid is essential. The data prescribed in this investigation provide realistic information for development and analysis of models of this behavior. A numerical model was suggested as one possibility for the gas phase models.

The data and numerical model show good agreement in the low surface temperature range studied. Prior to significant gas phase reactions it is expected that higher surface temperatures and heating rates will alter these results only in terms of gas phase boundary conditions. If these higher temperature preignition data become available, and a suitable model of detailed solid fuel pyrolysis is constructed, a complete model may be constructed, describing the sequence of fuel heating and pyrolysis, leading to detailed spatially and temporally resolved ignition predictions. It is hoped that these data and modeling will promote predictive abilities in the study of pyrolysis and ignition phenomena.

\section{APPENDIX}

A model was developed and a numerical procedure utilized to obtain solutions to the equations of conservation of mass, momentum, energy, and species concentration. The analysis incorporated the following assumptions:

1. The flow is considered a two-dimensional laminar natural convection boundary layer adjacent to a semi-infinite surface.
2. The boundary layer fluid is composed of a dilute binary mixture of air and vapor. The vapor is injected from the surface.

3. The surface temperature varies slowly with respect to the time required for the boundary layer variables to reach steady state values. Consequently, the surface temperature and the surface concentration of vapor are taken to be constant, and the steady state values of the numerical solution are used.

4. The temperature of the vapor injected into the boundary layer is the same as the surface temperature.

5. The thermal and concentration boundary layer thicknesses are approximately the same, i.e., Lewis number $(L e) \cong 1$,

6. The Soret and Dufour effects are negligible.

These assumptions are not expected to place severe restrictions on the applicability of the model; however, some discussion, particularly of assumption 5 , may be useful.

The Lewis number $(D / \alpha)$ is assumed of order one in order to simplify the energy equation [26] . Physically the simplification corresponds to neglecting heat transfer due to enthalpy flow caused by concentration gradients. This assumption appears physically reasonable if the thermal and concentration boundary layers are of approximately the same thickness, so that enthalpy flow across the boundary layer due to concentration gradients is small. Relative boundary layer thickness may be modeled as $(L e)^{1 / 3}$, by analogy with the $(P r)^{1 / 3}$ comparisons of thermal and momentum boundary layers. Thus for $(L e)^{1 / 3} \cong 1$ the above assumption holds and the following formulation is appropriate. In this calculation, the individual fluid properties are allowed to vary, while for any mixture the Schmidt number is held constant. In all the cases modeled, as for most vapor mixtures [26], the resulting $(L e)^{\mathbf{1} / 3} \cong 1$ requirement is met.

The equation corresponding to these assumptions are

$$
\frac{\partial \rho}{\partial t}+\frac{\partial(\rho u)}{\partial x}+\frac{\partial(\rho v)}{\partial y}=0
$$


$\frac{\partial u}{\partial t}+u \frac{\partial u}{\partial x}+v \frac{\partial u}{\partial y}$

$$
\begin{aligned}
& =g \beta_{t}\left(T-T_{\infty}\right)+g \beta_{c}\left(c-c_{\infty}\right) \\
& +\frac{1}{\rho} \frac{\partial}{\partial y} \mu \frac{\partial u}{\partial y},
\end{aligned}
$$

$\frac{\partial T}{\partial t}+u \frac{\partial T}{\partial x}+v \frac{\partial T}{\partial y}=\frac{1}{\rho c_{\mathrm{p}}} \frac{\partial}{\partial y} k \frac{\partial T}{\partial y}$,

$\frac{\partial c}{\partial t}+u \frac{\partial c}{\partial x}+v \frac{\partial c}{\partial y}=\frac{\partial}{\partial y} D \frac{\partial c}{\partial y}$,

where $x$ is in the vertical direction and $y$ is the direction normal to the surface. The other symbols are defined in the nomenclature. The properties were taken to be those of pure air, at the local temperature, giving [18]

$\rho=\frac{R T}{P}, \quad C_{\mathrm{p}}=C_{\mathrm{p}_{0}}\left(1+A\left(T-T_{0}\right)\right)$,

$k=k_{0}\left(1+M\left(T-T_{0}\right)\right), \quad \mu=\mu_{0}\left(1+C\left(T-T_{0}\right)\right)$,

$\beta_{\mathbf{T}}=\beta_{0}\left(1+B\left(T-T_{0}\right)\right)$,

$\beta_{c}=\frac{M_{1}-M_{2}}{c\left(M_{1}-M_{2}\right)+M_{2}}$.

The initial conditions for a specimen of semiinfinite vertical extent are

$u=0, \quad \rho=\rho_{\infty} \quad$ for $0 \leqslant x, 0 \leqslant y \leqslant \infty, t \leqslant 0$,

$v=0, \quad c=c_{\infty}, \quad T=T_{\infty}$

$$
\text { for } 0 \leqslant x, \quad 0<y \leqslant \infty, \quad t \leqslant 0 .
$$

The boundary conditions at the surface of the plate are

$-k \frac{\partial T}{\partial y}+\rho_{\mathrm{w}} V_{\mathrm{w}} C_{\mathrm{p}_{\mathrm{w}}} T=\rho_{\mathrm{s}} V_{\mathrm{s}} C_{\mathrm{p}_{\mathrm{s}}} T$

$-\rho D \frac{\partial c}{\partial y}+\rho_{\mathrm{w}} V_{\mathrm{w}} C_{\mathrm{w}}=\rho_{\mathrm{s}} V_{\mathrm{s}}$

$u=0$.
The first two of these conditions must be simplified for use in this model. The velocity of vapor in the solid, $V_{s}$, is not known, nor can it be calculated. Consequently, these two equations, valid at the solid-air mixture interface, must be simplified. In a finite difference sense, the interface corresponds to an element of vanishing size as $d y \rightarrow 0$. In order for the surface gradients to remain finite, $d C$ and $d T$ must also vanish. The resulting surface condition on the air mixture side of the interface can be given if a means of calculating $V_{\mathrm{w}}, C_{\mathrm{w}}$, and $T_{\mathrm{w}}$ is provided. Without details of the solid behavior, this calculation is impossible. Consequently, the boundary conditions at the solid interface used in this model will be the values of $V_{\mathrm{w}}, C_{\mathrm{w}}$, and $T_{\mathrm{w}}$ specified on the fluid side of the interface:

$$
\begin{aligned}
& v=v_{\mathrm{w}}(x), \quad T=T_{\mathrm{w}}(x), \quad c=c_{\mathrm{w}}(x), \\
& u=0 \quad 0 \leqslant x, \quad y=0 .
\end{aligned}
$$

At the leading edge of the plate,

$$
\begin{gathered}
u=0, \quad v=0, \quad c=c_{\infty}, \\
T=T_{\infty}, \quad x=0, \quad 0<y .
\end{gathered}
$$

Far from the plate,

$u=0, \quad v=0, \quad c=c_{\infty}, \quad T=T_{\infty}, \quad 0 \leqslant x, \quad y \rightarrow \infty$.

The calculation requires a knowledge of the parameters $c_{\mathbf{w}}$ and $v_{\mathrm{w}}$, which depend on the rate of vapor formation in, and emission from, the solid. Unfortunately, the vapor formation and emission processes depend strongly on empirical factors such as pitting, checking, drying, and cracking of the materials. At the present time, there are no suitable models available, that would yield $c_{\mathrm{w}}$ and $v_{\mathrm{w}}$ with high accuracy for the materials used.

To circumvent this difficulty in the calculations, the $c_{\mathrm{w}}$ value provided by the data was used. To obtain $c_{\mathrm{w}}$, the concentration values available in the boundary layer, were extrapolated to the wall. The injection velocity $v_{\mathrm{w}}$ was estimated by applying conservation of mass to each species 
in a small control volume placed adjacent to the wall. This gives [9]

$v_{\mathrm{w}}=\frac{1.28 c_{\mathrm{w}}}{1-c_{\mathrm{w}}}$

The validity of this procedure for estimating $v_{\mathrm{w}}$ was checked with propane injected through a sintered bronze plate. In this situation both $v_{\mathrm{w}}$ and $c_{\mathrm{w}}$ were measured independently. The measured $v_{\mathrm{w}}$ values agreed to within $\sim 10 \%$ with those calculated from Eq. (A.10).

Equations (A.1)-(A.10) were nondimensionalized and solved using an explicit finite difference scheme similar to that of Carnahan et al. [19]. Care needs to be taken to select small enough temporal step sizes to ensure stability of the numerical method. The approximate criterion for $\operatorname{Pr} \cong 1$ is

$\Delta t \leqslant \frac{\beta_{\mathrm{T}}\left(T_{\mathrm{w}}-T_{\infty}\right)}{\beta_{\mathrm{c}}\left(c_{\mathrm{w}}-c_{\infty}\right)}\left[\frac{u}{\Delta x}+\frac{v}{\Delta y}+\frac{1}{S c} \frac{2}{(\Delta y)^{2}}\right]^{-1}$,

where the variables are described in the nomenclature. This requires either extremely small $\Delta t$, where $u$ and $v$ are small, or a variable step size $(\Delta x)$. The latter route was chosen here, allowing the step size to vary according to Eq. (A.11). Numerical convergence was tested by halving the geometric step sizes (with appropriate changes in $\Delta t$ ) and independently halving $\Delta t$. The variation between the results was less than $0.1 \%$, which was considered sufficient. Further details of the application of the numerical method are given in [9].

The boundary layer equations (A.1)-(A.4) do not hold either for small elapsed times or near the leading edge of the specimen. Since the flow exists in what amounts to a favorable pressure gradient, the use of the boundary layer assumptions in these ranges should not significantly affect solutions at long times far from the leading edge (i.e., favorable gradients typically damp out upstream history). As noted in assumption 3 , the steady state solution is utilized and has been compared with previous solutions in two cases, as noted below.
The accuracy of the model and numerical method were tested by comparing results obtained by the model with previous solutions. Steady state solutions were compared with ones developed by Ostrach [10] (Fig. 2) and Eichhorn [20] In each case, with the appropriate boundary conditions, the steady state values calculated by the current method were in good agreement with the previous results. The transient solutions were checked in several ways. For the case of pure thermal convection, transient temperature and velocity profiles were compared with Hellums [21] and the expected conduction-type solution for the early profiles [22]. Combined thermal and concentration buoyancy induced transient solutions were compared with those of Callahan and Marner [17]. In each case, the agreement was better than $1.0 \%$ in the values calculated for each of the thermal, concentration, and velocity profiles.

\section{NOMENCLATURE}

$A \quad$ molar refractivity, $\mathrm{m}^{3} /$ mole

$C_{\mathrm{p}} \quad$ specific heat of the fluid at constant pressure, $\mathrm{J} / \mathrm{kg} \mathrm{K}$

$c \quad$ molar concentration of component 1 in a binary mixture, moles/mole

$D$ molar diffusivity of the fluid, $\mathrm{m}^{2} / \mathrm{s}$

$k$ thermal conductivity of the fluid, $\mathrm{W} / \mathrm{m} \mathrm{K}$

Le $\quad$ Lewis number, $D K^{-1}$

$l$

$M$

path length traversed by object beam, $m$

molecular weight, $\mathrm{kg}$ mole $/ \mathrm{mole}$

$\dot{m}_{\mathrm{w}} \quad$ mass flow rate of component 1 (vapor) at the specimen surface, $\mathrm{kg} / \mathrm{s}$

Prandtl number, $\nu k^{-1}$ measured phase shift, degrees universal gas constant, $\mathrm{J} /$ mole $\mathrm{K}$ number of moles of a given component present in the mixture, moles

$S \quad$ fringe number

$S_{c} \quad$ Schmidt number, $\nu D^{-1}$

$T \quad$ temperature of the fluid, ${ }^{\circ} \mathrm{C}$

$u \quad$ velocity component in the $x$ or vertical direction

velocity component in the $y$ or horizontal direction 
$x \quad$ vertical coordinate

$y$ horizontal coordinate

$\alpha \quad$ thermal diffusivity, $\mathrm{m}^{2} / \mathrm{s}$

$\beta_{c}$ expansion coefficient due to concentration, moles/mole

$\beta_{\mathrm{T}}$ expansion coefficient due to temperature, $1 /^{\circ} \mathrm{C}$

$\lambda \quad$ wavelength of laser light, $m$

$\mu \quad$ absolute viscosity of the fluid, $\mathrm{N} \mathrm{s} / \mathrm{m}^{2}$

$\nu \quad$ kinematic viscosity of the fluid, $\mathrm{m}^{2} / \mathrm{s}$

$\nu_{\alpha}, \nu_{\beta}, \nu_{\gamma}$ coefficients for numbers of molecules of type $\alpha, \beta$, and $\gamma$

$\rho$ density of the fluid, $\mathrm{kg} / \mathrm{m}^{3}$

\section{Subscripts}

w variable evaluated at specimen surface

$\infty \quad$ variable evaluated in pure air at ambient temperature and pressure

1 component 1 of the binary mixture (vapor)

2 component 2 of the binary mixture (air)

\section{REFERENCES}

1. Martin, S., Tenth Symposium (International) on Combustion, 1965, pp. 877-896.

2. Kung, H.C., Combust. Flame 18:185-195 (1972).

3. Kashiwagi, T., Combust. Sci. Technol. 8:225-236 (1974).

4. Ndubizu, C. C., and Durbetaki, P., Fire Research 1:281-290 (1977).

5. Kung, H.-C., Fifteenth Symposium (International) on Combustion, 1974, pp. 243-253.

6. Farrell, P. V., Springer, G. S., and Vest, C. M., $A p$ plied Optics 21:1624-1627 (1982).

7. Ostrach, S., Physico Chemical Hydrodynamics $1: 233-$ 247 (1980).

8. Stamm, A. J., Ind. and Eng. Chem. 48:413-417 (1956).
9. Farrell, P. V., Ph.D. Thesis, University of Michigan, 1982.

10. Ostrach, S., NACA Report 1111, pp. 63-79, 1953.

11. Dandliker, R., in Progress in Optics XVII (E. Wolf, Ed.), North-Holland, Amsterdam, 1980, pp. 2-84.

12. Born, M., and Wolf, E., Principles of Optics, 6 th Ed., Pergamon Press, New York, 1980, pp. 87-92.

13. Cha, S., and Vest, C. M., Applied Optics 20:27872794 (1981).

14. Hauf, W., and Grigull, U., in Advances in Heat Transfer 6 (J. P. Hartnett and T. F. Irvine, Jr., Eds.), Academic Press, New York, 1969, pp. 265-266.

15. Kothandraman, C. P., and Subramanyan, S., Heat and Mass Transfer Data Book, 3rd Ed., Wiley, New York, 1977, pp. 144-147.

16. Gebhart, B., and Pera, L., Int. H. Heat and Mass Transfer 14:2025-2050 (1971).

17. Callahan, G. D., and Marner, W. J., Int. J. Heat and Mass Transfer 19:165-174 (1976).

18. Gray, D. D., and Giorgini, A., Int. J. Heat and Mass Transfer 19:545-551 (1976).

19. Carnahan, B., Luther, H., and Wilkes, J., Applied Numerical Methods, Wiley, New York, 1969, pp. 474-481.

20. Eichhorn, R., J. Heat Transfer 82:260-263 (1960).

21. Hellums, J. D., Ph.D. Thesis, University of Michigan, 1960.

22. Siegel, R., Trans. Am. Soc. Mech. Engr. 80:347-359 (1958).

23. International Critical Tables, McGraw-Hill, New York, 1933, pp. 6-15.

24. Williams, F. A., in Heat Transfer in Fires: Thermo. Physics, Social Aspects, and Economic Aspects (P. L. Blackshear, Ed.), Scripta, New York, 1974, pp. 197237.

25. Ibid., pp. 231-233.

26. Kays, W. M., Convective Heat and Mass Transfer, McGraw-Hill, New York, 1966, pp. 302-303.

27. Gardiner, W. C., Hidaka, Y., and Tanzawa, T., Combust. Flame 40:213-219 (1981).

Received 23 November 1982; revised 14 April 1983 